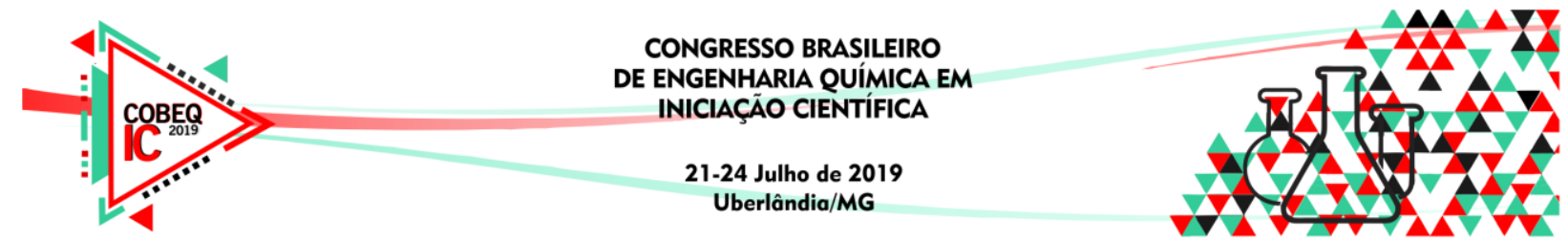

\title{
ADSORÇÃO DO ÁCIDO 1,4-CICLOHEXANODICARBOXÍLICO EM CARVÃO ATIVADO
}

\author{
L. R. R. LIMA ${ }^{1}$, J. D. da SILVA ${ }^{1}$ e I. C. OSTROSKI ${ }^{1}$ \\ ${ }^{1}$ Universidade Federal de Goiás, Departamento de Engenharia Química \\ E-mail para contato: laressarayane@ hotmail.com; jonasgabriel0599@yahoo.com.br; \\ indianara_ostroski@ufg.br
}

\begin{abstract}
RESUMO - Os ácidos naftênicos juntamente com compostos sulfurosos e/ou nitrogenados são os principais contaminantes do petróleo e de sua água residual, e trazem sérios problemas ao meio ambiente e ao processo de refino. Assim, este trabalho teve como objetivo avaliar a capacidade adsortiva do carvão ativado de osso de boi, com e sem tratamento básico, para a remoção do ácido naftênico 1,4ciclohexanodicarboxílico. No estudo cinético, as duas curvas se ajustaram melhor ao modelo de pseudo segunda ordem, sendo que o carvão tratado chegou ao equilíbrio mais rápido que o carvão sem o tratamento. Os dados de equilíbrio foram ajustados aos modelos de Langmuir e Freundlich, ambos apresentaram um bom ajuste. A partir da análise da constante de equilíbrio, o carvão com tratamento apresenta uma maior afinidade pelo adsorvato e a quantidade retida de ácido, obtido experimentalmente, foi de $381,78 \mathrm{mg} \mathrm{g}^{-1}$.
\end{abstract}

\section{INTRODUÇÃO}

A indústria petroquímica é uma grande emissora de poluição. Dentre derramamento de óleos, emissões de óxidos de enxofre e hidrogênio, está um resíduo da etapa de extração, a água residuária oleosa (Mariano, 2001). Atualmente esse resíduo é tratado por coagulação, biodigestão ou o uso de membrana separadora (Yu, 2017). Para remoção de compostos dissolvidos que podem conferir acidez acentuada às águas, técnicas como remoção líquidolíquido (Anderson et al.,2013), adsorção em espumas de carbono (Yang et al., 2019) ou adsorção em materiais com alta área superficial, tal como carvão ativado (Caixeta, 2016) podem ser utilizados.

Estudos têm mostrado que esses ácidos dissolvidos, mesmo em baixas concentrações na água são prejudiciais a diversos organismos (Martinez-Iglesias et al., 2015). Um dos ácidos naftênicos presente nestas águas é o ácido 1,4-ciclohexanodicarboxílico $\left(\mathrm{C}_{8} \mathrm{H}_{12} \mathrm{O}_{4}\right)$. Sua remoção pode ser feita em um processo de adsorção. Para tanto foi realizado um estudo da cinética e do equilíbrio de adsorção em carvão ativado de osso de boi. Além do carvão bruto, o mesmo foi submetido a um tratamento básico com intento de se melhorar o processo.

\section{METODOLOGIA}

\subsection{Tratamento do carvão ativado}




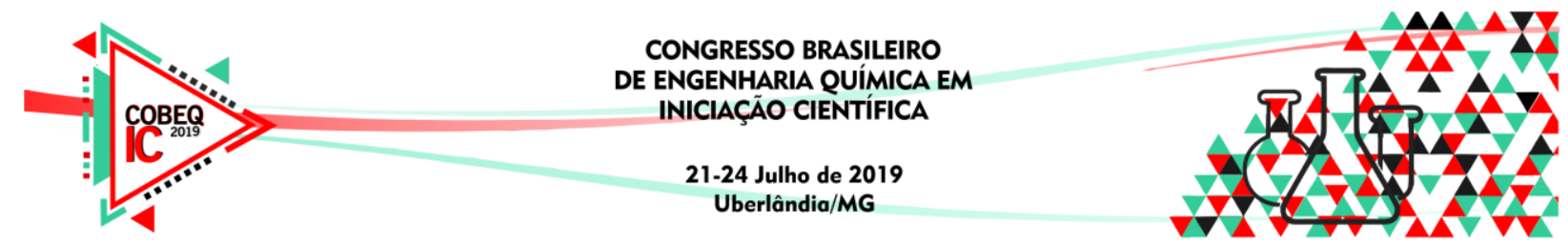

Inicialmente foi realizada a análise granulométrica do carvão ativado comercial (Bonechar Carvão Ativado do Brasil). Em seguida, o mesmo foi tratado com $\mathrm{NaOH}$ seguindo o procedimento descrito por Chiang et al. (2002). Foram pesados $10 \mathrm{~g}$ do adsorvente, em que tal quantidade foi colocada em erlenmeyers de $125 \mathrm{ml}$. Em seguida, a cada erlenmeyer, foi adicionado $50 \mathrm{ml}$ de solução de hidróxido de sódio $(\mathrm{NaOH})$ 2,0 mols. $\mathrm{L}^{-1}$. Dessa forma, as misturas foram colocadas em um shaker e mantidas sob agitação de $120 \mathrm{rpm}$ por 24 horas, a $25^{\circ} \mathrm{C}$. Após esse período, as amostras de carvão ativado foram filtradas e lavadas com água, depois submetidas a um tratamento térmico à $130^{\circ} \mathrm{C}$ por 48 horas.

\subsection{Determinação da cinética de adsorção}

Para determinar a cinética de adsorção, foram pesados $50 \mathrm{mg}$ do carvão de osso de boi não tratado, esta quantidade foi inserida em 11 erlenmeyers de $125 \mathrm{ml}$. Em seguida, foi adicionado em cada um dos erlenmeyers, $30 \mathrm{ml}$ de uma solução aproximadamente de 1 g.L.-1 de ácido 1,4-ciclohexanodicaboxílico, os mesmos foram colocados imediatamente em um shaker, com a agitação de $145 \mathrm{rpm}$ e $30^{\circ} \mathrm{C}$. As amostras foram retiradas nos seguintes intervalos de tempo: 5, 10, 15, 20,30,60,120, 240, 360, 480 e 720 min, e filtrados à vácuo. As soluções, isentas de carvão, foram tituladas com $\mathrm{NaOH} 0,0045 \mathrm{M}$ para a determinação da quantidade remanescente de ácido naftênico na solução. Este mesmo processo foi realizado para o carvão tratado.

\subsection{Determinação da isoterma de adsorção}

Para a obtenção dos dados experimentais de equilíbrio, foram preparadas soluções de ácido naftênico, com as seguintes concentrações em g.L. $\mathrm{L}^{-1}: 0,95 ; 1,15 ; 1,34 ; 1,52 ; 1,7 ; 1,93$. Estas concentrações foram determinadas a partir da titulação com $\mathrm{NaOH}$. Cada uma dessas soluções foi adicionada a dois erlenmeyers, um contendo carvão tratado e outro contendo não tratado, e todas as amostras foram levadas ao shaker, permanecendo na agitação de $145 \mathrm{rpm}$ e $30^{\circ} \mathrm{C}$ por 6 horas.

Como houve flutuações durante o procedimento experimental, alguns pontos foram retirados das curvas cinéticas e das isotermas. Experimentos futuros serão realizados para obtenção destes dados.

\subsection{Equacionamento}

A seguir são apresentados os modelos utilizados para análise dos dados cinéticos e de equilíbrio. Modelos cinéticos de pseudo primeira e pseudo segunda ordem, e os modelos de equilíbrio de Langmuir e de Freundlich na forma das equações 1, 2, 3 e 4, respectivamente.

$$
\begin{aligned}
& \ln \left(\mathrm{q}_{\mathrm{a}}-\mathrm{q}_{\mathrm{t}}\right)=\ln \mathrm{q}_{\mathrm{a}}-\mathrm{k}_{1} \mathrm{t} \\
& \frac{1}{\mathrm{q}_{\mathrm{t}}}=\frac{1}{\mathrm{q}_{\mathrm{g}}}+\frac{1}{\mathrm{k}_{2} \mathrm{q}_{\mathrm{a}}^{2}} * \frac{1}{\mathrm{t}} \\
& \frac{1}{\mathrm{q}_{\mathrm{g}}}=\frac{1}{\mathrm{q}_{\max }}+\frac{1}{\mathrm{q}_{\max } \mathrm{K}_{\mathrm{L}}} * \frac{1}{\mathrm{c}_{\mathrm{g}}}
\end{aligned}
$$




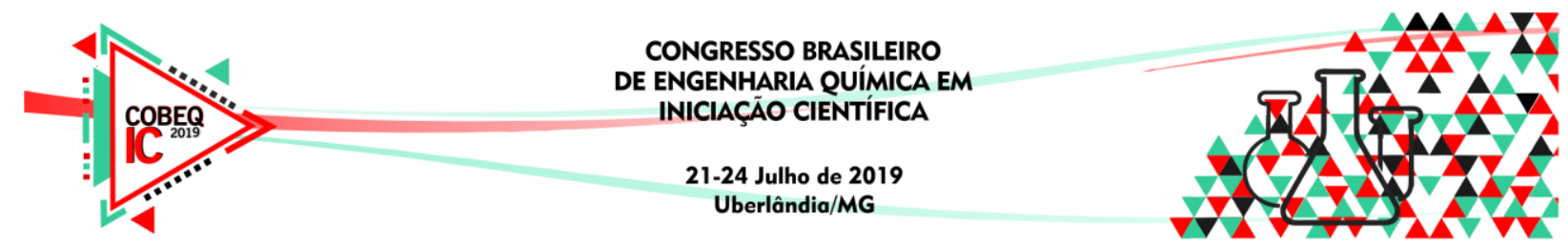

$$
\ln \mathrm{q}_{\mathrm{a}}=\ln \mathrm{K}_{\mathrm{f}}+\frac{1}{\mathrm{n}} \ln \mathrm{C}_{\mathrm{e}}
$$

Em que, para todas as equações, $\mathrm{q}_{\mathrm{e}}, \mathrm{q}_{\mathrm{t}}, \mathrm{k}_{1}, \mathrm{t}, \mathrm{k}_{2}, \mathrm{q}_{\mathrm{max}}, \mathrm{K}_{\mathrm{L}}, \mathrm{C}_{\mathrm{e}}, 1 / \mathrm{n}$ e $\mathrm{K}_{\mathrm{f}}$ são respectivamente, quantidade de soluto adsorvido por grama de adsorvente no equilíbrio $\left(\mathrm{mg} \cdot \mathrm{g}^{-1}\right)$, quantidade de soluto adsorvido por grama de adsorvente no tempo $\mathrm{t}\left(\mathrm{mg} \cdot \mathrm{g}^{-1}\right)$, constante de velocidade de adsorção de pseudo primeira ordem $\left(\min ^{-1}\right)$, tempo $(\mathrm{min})$, constante de velocidade de adsorção de pseudo segunda ordem $\left(\mathrm{g} \cdot \mathrm{mg}^{-1} \cdot \mathrm{min}^{-1}\right)$, quantidade de soluto adsorvida por grama de adsorvente máxima (mg. $\left.\mathrm{g}^{-1}\right)$, constante de adsorção de Langmuir $\left(\mathrm{L}_{\mathrm{mg}} \mathrm{m}^{-1}\right)$, concentração do soluto de equilíbrio (g. $\left.\mathrm{L}^{-1}\right)$, parâmetro empírico adimensional de Freundlich e constante de adsorção de Freundlich $\left(\left(\mathrm{L}_{\mathrm{m}} \mathrm{mg}^{-1}\right)^{1 / \mathrm{n}}\left(\mathrm{mg} \cdot \mathrm{g}^{-1}\right)\right)$. Os valores de $\mathrm{q}_{\mathrm{e}}$ foram obtidos a partir da equação 5 .

$$
\mathrm{q}_{\mathrm{a}}=\frac{\left(\mathrm{C}_{0}-\mathrm{C}_{\mathrm{e}}\right) \mathrm{v}}{\mathrm{m}_{\mathrm{v}}}
$$

Em que $\mathrm{C}_{0}\left(\mathrm{mg} . \mathrm{L}^{-1}\right)$ é a concentração inicial do adsorvato, $\mathrm{C}_{\mathrm{e}}\left(\mathrm{mg} \cdot \mathrm{L}^{-1}\right)$ é a concentração do adsorvato no equilíbrio, $\mathrm{m}_{\mathrm{c}}(\mathrm{g})$ é a massa do adsorvente e $\mathrm{V}(\mathrm{L})$ é o volume da solução.

\section{RESULTADOS}

\subsection{Estudo Cinético}

Nos estudos cinéticos foi observado que para o carvão sem tratamento químico, o sistema entrou em equilíbrio em aproximadamente 6 horas com qe de $159,89 \mathrm{mg} . \mathrm{g}^{-1}$ e para o carvão tratado, o sistema entrou em equilíbrio em aproximadamente 4 horas com q de 244,72 mg. $\mathrm{g}^{-1}$. Os dados experimentais foram ajustados aos modelos de pseudo primeira e pseudo segunda ordem, e os resultados são apresentadas na Figura 1 e os parâmetros cinéticos estão apresentados na Tabela 1.

Figura 1 - Cinética do carvão sem tratamento (esquerda) e com tratamento (direita) com os ajustes não lineares dos modelos de pseudo primeira ordem e pseudo segunda ordem.
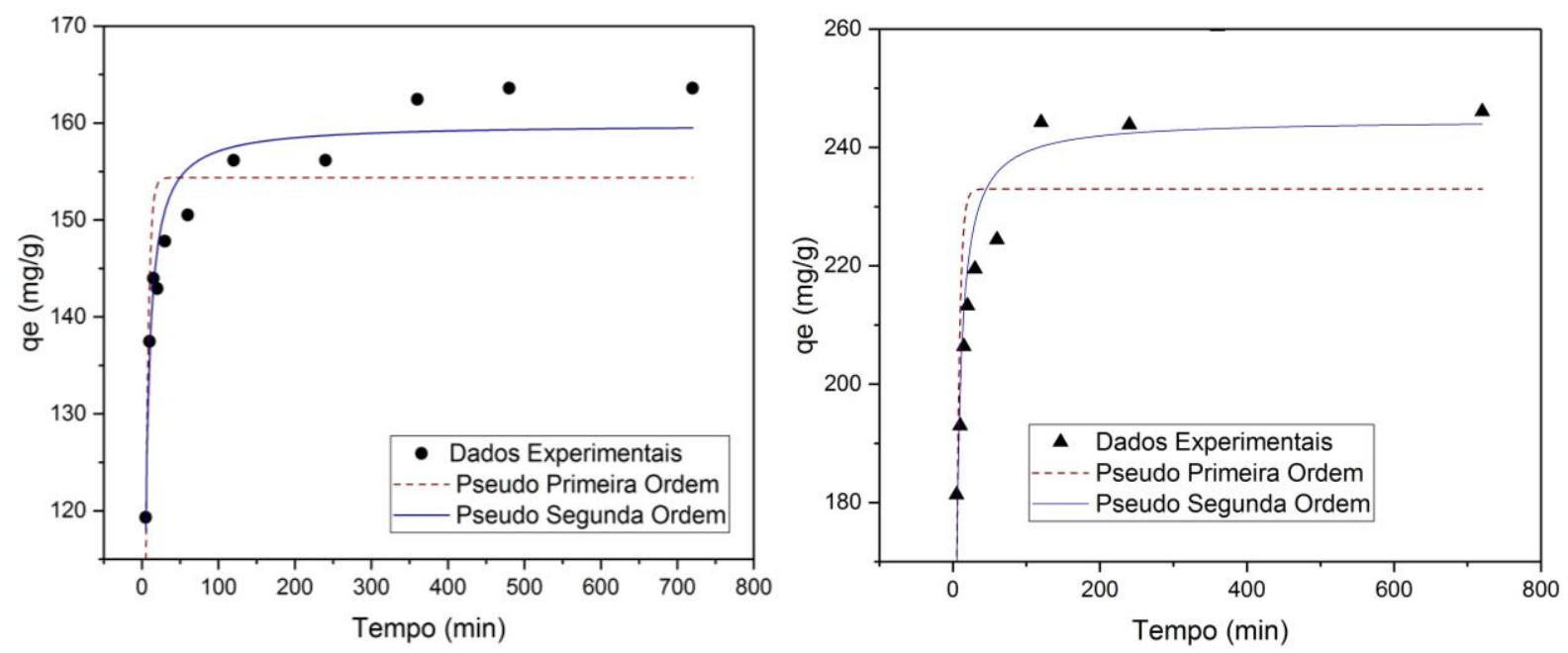


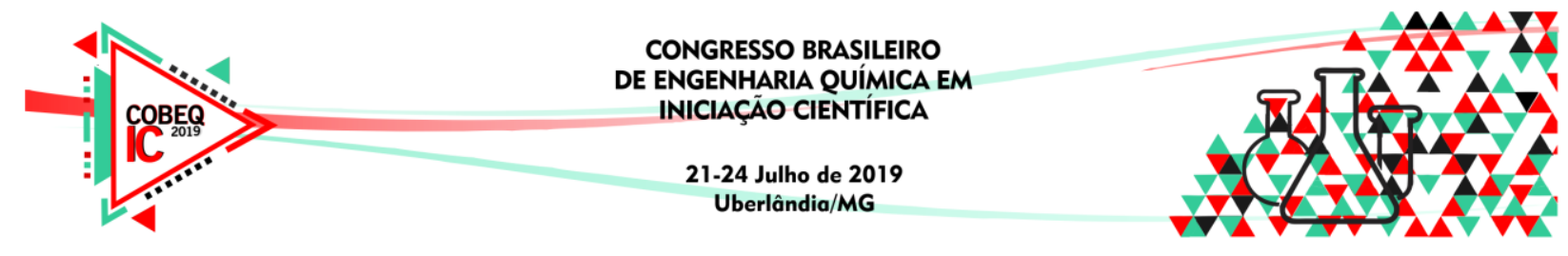

Tabela 1 - Parâmetros cinéticos calculados para os modelos de pseudo primeira e segunda ordem nos carvões ativados de osso de boi tratado e não tratado

\begin{tabular}{|c|c|c|c|}
\hline Modelo Cinético & Parâmetros & $\begin{array}{c}\text { Carvão Ativado Osso } \\
\text { de Boi Não Tratado }\end{array}$ & $\begin{array}{c}\text { Carvão Ativado Osso } \\
\text { de Boi Tratado }\end{array}$ \\
\hline \hline \multirow{3}{*}{ Pseudo Primeira Ordem } & $\mathrm{q}_{\mathrm{e}}\left(\mathrm{mg} \cdot \mathrm{g}^{-1}\right)$ & $154,38 \pm 2,64$ & $232,98 \pm 6,93$ \\
\cline { 2 - 4 } & $\mathrm{K}_{1}\left(\mathrm{~min}^{-1}\right)$ & $0,27 \pm 0,04$ & $0,24 \pm 0,05$ \\
\cline { 2 - 4 } & $\mathrm{R}^{2}$ & 0,6973 & 0,5133 \\
\hline \multirow{3}{*}{ Pseudo Segunda Ordem } & $\mathrm{q}_{\mathrm{e}}\left({\left.\mathrm{mg} \cdot \mathrm{g}^{-1}\right)}^{-1}\right)$ & $159,89 \pm 1,42$ & $244,72 \pm 4,54$ \\
\cline { 2 - 4 } & $\mathrm{K}_{2}\left(\mathrm{~g} \cdot \mathrm{mg}^{-1} \cdot \mathrm{min}^{-1}\right)$ & $0,0035 \pm 0,0004$ & $0,0018 \pm 0,0003$ \\
\cline { 2 - 4 } & $\mathrm{R}^{2}$ & 0,9390 & 0,8622 \\
\hline
\end{tabular}

A partir da Tabela 1, é possível observar que o modelo de pseudo segunda ordem foi o que melhor se ajustou aos dados experimentais para os dois sistemas. A expressão de taxa de pseudo segunda ordem normalmente é utilizada para descrever a quimissorção envolvendo forças de valência por meio do compartilhamento ou troca de elétrons entre o adsorvente e o adsorvato como forças covalentes e troca iônica. A vantagem de usar este modelo é que não há necessidade de conhecer a capacidade de equilíbrio dos experimentos, pois ele pode ser calculado a partir do modelo (Ho, 2006a).

\subsection{Estudo de Equilíbrio}

Para o estudo da adsorção no equilíbrio, foram utilizados dois modelos Langmuir e Freundlich. É preferível, assim, utilizar a equação não linearizada, pois há discordâncias nos valores das constantes obtidas quando comparada a equação linearizada (Ho, 2006b). As isotermas de adsorção do carvão de osso de boi tratado e não tratado estão apresentadas na Figura 2. Os parâmetros encontrados a partir dos dados experimentais; bem como, os valores dos ajustes dos modelos estão dispostos na Tabela 2.

Figura 2 - Isoterma de adsorção com os ajustes não lineares dos modelos de Langmuir e

Freundlich para o carvão de osso de boi não tratado (esquerda) e tratado (direita).
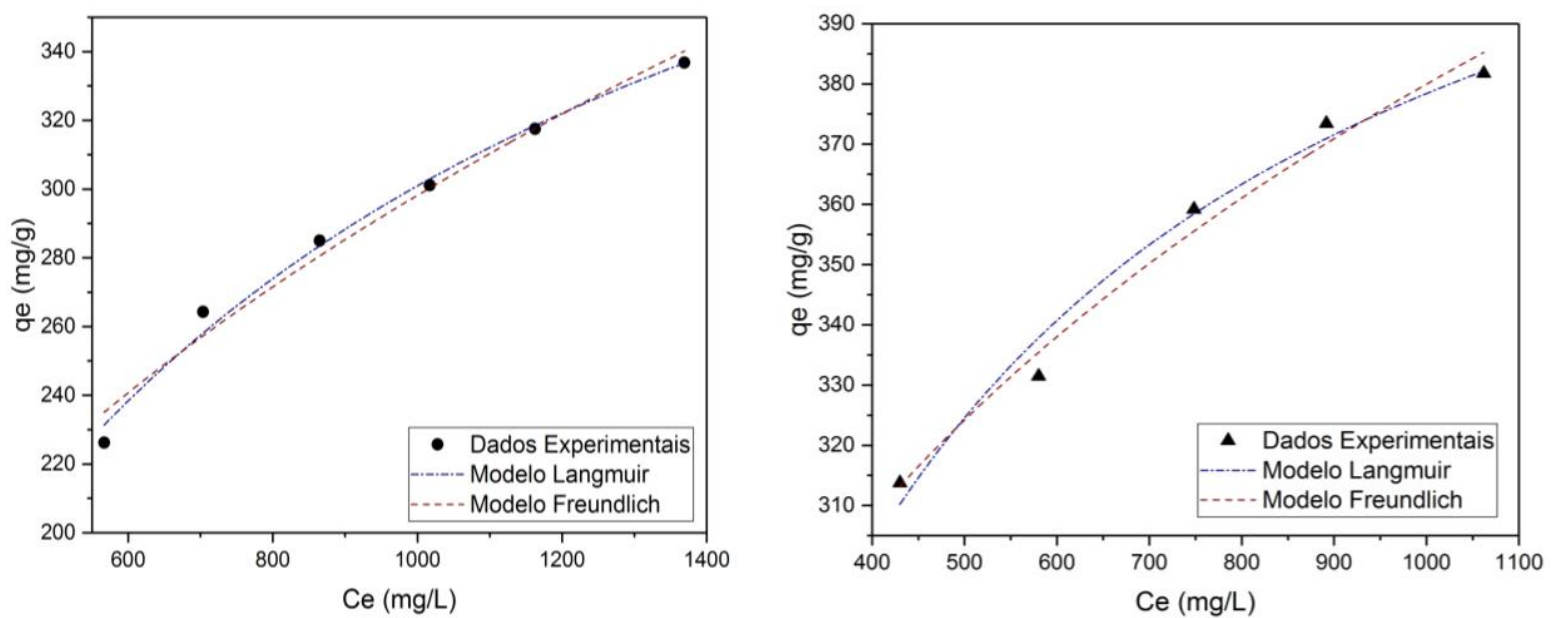


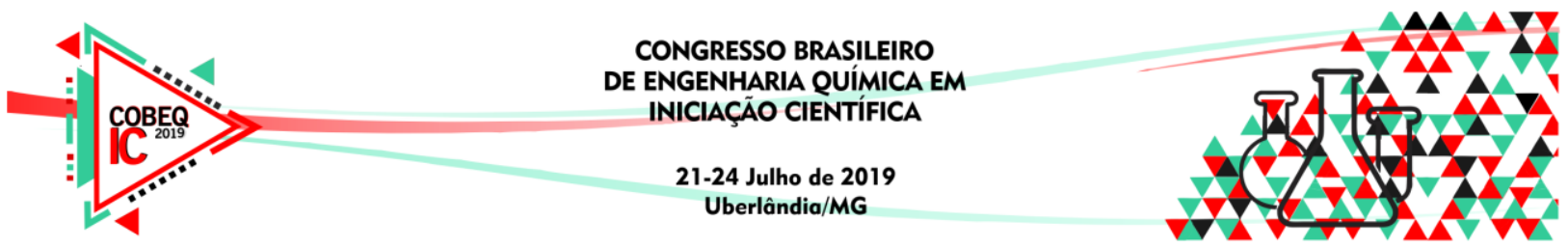

Tabela 2 - Parâmetros das isotermas de adsorção do ácido 1,4-ciclohexanodicarboxílico com carvão de osso de boi tratado e não tratado

\begin{tabular}{|c|c|c|c|}
\hline Modelo & Parâmetros & $\begin{array}{c}\text { Carvão Ativado Osso } \\
\text { de Boi Não Tratado }\end{array}$ & $\begin{array}{c}\text { Carvão Ativado } \\
\text { Osso de Boi Tratado }\end{array}$ \\
\hline \hline \multirow{3}{*}{ Langmuir } & $\mathrm{k}_{\mathrm{L}}\left(\mathrm{L} \cdot \mathrm{mg}^{-1}\right)$ & $0,0015 \pm 0,0001$ & $0,0050 \pm 0,0005$ \\
\cline { 2 - 4 } & $\mathrm{Q}_{\max }\left(\mathrm{mg}^{-1} \mathrm{~g}^{-1}\right)$ & $496,39 \pm 17,57$ & $453,71 \pm 10,68$ \\
\cline { 2 - 4 } Freundlich & $\mathrm{R}^{2}$ & 0,9909 & 0,98164 \\
\cline { 2 - 4 } & $\mathrm{k}_{\mathrm{F}}\left(\mathrm{L} \cdot \mathrm{g}^{-1}\right)$ & $16,49 \pm 3,54$ & $78,18 \pm 8,86$ \\
\cline { 2 - 4 } & $\mathrm{n}$ & $2,39 \pm 0,18$ & $4,37 \pm 0,33$ \\
\cline { 2 - 4 } & $\mathrm{R}^{2}$ & 0,9798 & 0,9841 \\
\hline
\end{tabular}

Analisando os coeficientes de $\mathrm{R}^{2}$ na Tabela 2, para o carvão não tratado, o modelo de Langmuir aparece com um melhor ajuste aos dados experimentais, no caso do carvão com tratamento, o melhor ajuste foi no modelo de Freundlich. Seus valores dos coeficientes de determinação, entretanto, estão muito próximos; dessa forma para uma avaliação mais verossímil e crível, de qual modelo se ajusta melhor as isotermas, seria necessário realizar o ensaio novamente com mais pontos experimentais.

Analisando as constantes de adsorção "k", o carvão tratado apresentou um valor três vezes maior que o não tratado, nos dois modelos. As constantes de adsorção estão relacionadas com a energia livre de adsorção e a afinidade entre a superfície do adsorvente e o adsorvato. Dessa maneira, percebe-se que o carvão de osso de boi tratado é uma melhor alternativa para a adsorção desse ácido naftênico (Nascimento et al., 2014).

No estudo feito por Martinez-Iglesias et al. (2015) a partir da remoção do ácido 1,4ciclohexanodicarboxílico com um carvão ativado comercial, a capacidade máxima adsortiva foi de $452,6 \mathrm{mg} \cdot \mathrm{g}^{-1}$ em um $\mathrm{pH}$ 4. Os resultados da Tabela 2 indicam valores um pouco maiores, comprovando assim, que o carvão em estudo é uma boa escolha para a adsorção do respectivo ácido. Ao se comparar o estudo da cinética e da isoterma de equilíbrio, houve discordâncias entre os dados na concentração de $1 \mathrm{~g} / \mathrm{L}$ no tempo de 6 horas, deste modo devese investigar esse erro a partir da realização de novos ensaios experimentais.

Os pesquisadores Winter et al. (2016) apresentaram a caracterização do carvão ativado de osso de boi bruto e com tratamento químico, sendo os resultados respectivamente: ambos classificado como mesoporosos (área superficial de 123,3 $\mathrm{m}^{2} \mathrm{~g}^{-1}, 83,6 \mathrm{~m}^{2} \mathrm{~g}^{-1}$ ), com concentração de $1,4 \mathrm{~m}_{\text {eq }} \mathrm{g}^{-1}$ e $2,0 \mathrm{~m}_{\mathrm{eq}} \mathrm{g}^{-1}$ de grupos básicos (quantificado pelo método de Boehm) e ponto de carga zero (PCZ) igual a 8,1 e 10,3. Segundo Apeel et al. (2003), em valores de $\mathrm{pH}$ inferior ao PCZ, a carga superficial desse adsorvente é positiva e a adsorção de ânions é favorecida. A caracterização demostra que com o tratamento, a polaridade da superfície do carvão aumenta, sendo assim, o mesmo torna-se melhor adsorvente para os ácidos naftênicos. Esses resultados são comprovados pela cinética de adsorção e pelo estudo de equilíbrio.

\section{CONCLUSÃO}

Diante da análise do carvão ativado de osso de boi tratado e não tratado na remoção do ácido 1,4-ciclohexanodicarboxílico, os carvões ativados demonstram ser boas escolhas como 


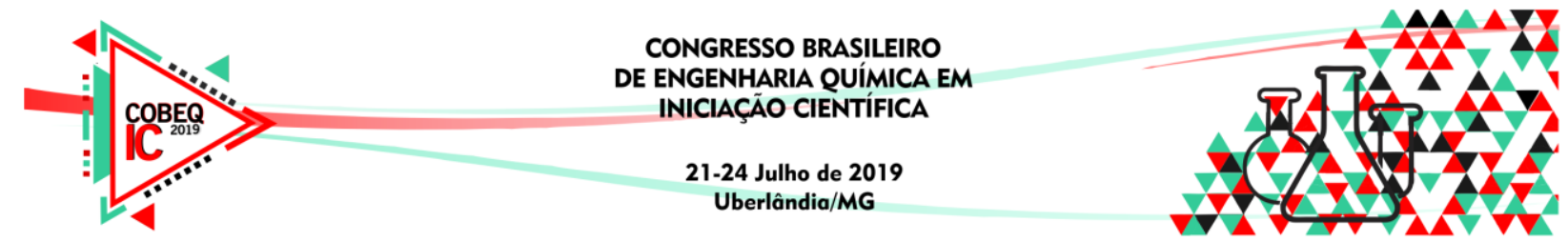

adsorvente. O estudo cinético constatou que após 4 horas o equilíbrio foi alcançado no sistema utilizando o carvão tratado, já com o não tratado, o tempo foi de 6 horas. No estudo de equilíbrio foram obtidos valores experimentais de máxima adsorção de 336,74 e 381,76 mg. $\mathrm{g}^{-1}$. Além disso, o carvão tratado apresentou uma constante de adsorção maior que o não tratado, portanto, é uma boa alternativa de adsorvente para este ácido naftênico.

\section{REFERÊNCIAS}

ANDERSON, K.; GOODRICH, P.; HARDACRE, C.; HUSSAIN, A.; ROONEY D W, WASSELL, D. Removal of naphthenic acids from crude oil using amino acid ionic liquids. Fuel, [s.1.], v. 108, p.715-722, jun. 2013.

APEEL, C.; MA, L. Q.; RHUEL, R. D; KENNELLEY, E.. Point of zero charge determination in soils and minerals via traditional methods and detection of electroacoustic mobility. Geoderma, v. 113, p. 77-93, abr. 2003.

CAIXETA, B. A. Remoção de Compostos Ácidos do Petróleo em Diferentes Tipos de Carvão Ativado. 2016. 78 f. Dissertação (Mestrado) - Curso de Química, Universidade Federal de Goiás, Goiânia, 2016.

CHIANG, H. L.; HUANG, C. P.; CHIANG, P.C. The surface characteristics of activated carbon as affected by ozone and alkaline treatment. Chemosphere, [s.1.], v. 47, n. 3, p.257-265, abr. 2002.

HO, Y. S. Review of second-order model for the sorption systems. J. Hazard. Mater., v. 136, p. 681-689, ago. 2006a.

$\mathrm{HO}, \mathrm{Y}, \mathrm{S}$. Isotherms for the sorption of lead onto peat: comparison of linear and nonlinearmethods. Pol. J. Environ. Stud., v. 15, n. 1, p. 81-86, jan. 2006 b.

MARIANO, J. B. Impactos Ambientais do Refino de Petróleo. Dissertação (Mestrado) Curso de Ciências em Planejamento Energético, Universidade Federal do Rio de Janeiro, Rio de Janeiro, 2001.

MARTINEZ-IGLESIAS, A.; NIASAR, S. H.; XU, C.; RAY, B. M. Adsorption of Model Naphthenic Acids in Water with Granular Activated Carbon. Adsorpt. Sci. Technol., [s.1.], v. 33, n. 10, p.881-894, dez. 2015.

NASCIMENTO, R. F.; LIMA, A. C. A.; VIDAL, C. B; MELO, D. Q.; RAULINO, G. S. C.; Adsorção aspectos teóricos e aplicações ambientais. $1^{\text {a }}$ ed. Ceará: Impressa Universitária, 2014

WINTER, C.; CAETANO, J. N.; ARAÚJO, A. B. C.; CHAVES, A. R.; OSTROSKI, I. C.; VAZ B. G.; PÉREZ, C. N.; ALONSO, C. G.. Activated carbons for chalcone production: Claisen-Schmidt condensation reaction. Chem. Eng. J., v. 303, p.604-610, jun. 2016.

YANG, S.; WANG, F.; TANGA, Q.; WANG, P.; XUP, Z. LIANG, J. Utilization of ultralight carbon foams for the purification of emulsified oil wastewater and their adsorption kinetics. Chem. Phys., [s.1.], v. 516, p.139-146, jan. 2019.

YU, L.; HAN, M.; HE, F.. A review of treating oily wastewater. Arab. J. Chem., [s.1.], v. 10, p.1913-1922, maio 2017. 